\title{
The role of multiple negative social relationships in inflammatory cytokine responses to a laboratory stressor
}

Sunmi Sunmi Song, Jennifer E Graham-Engeland, Elizabeth J Corwin, Rachel M Ceballos, Shelley E Taylor, Teresa Seeman, Laura Cousino Klein

The present study examined the unique impact of perceived negativity in multiple social relationships on endocrine and inflammatory responses to a laboratory stressor. Via hierarchical cluster analysis, those who reported negative social exchanges across relationships with a romantic partner, family, and their closest friend had higher mean IL-6 across time and a greater increase in TNF- $\alpha$ from $15 \mathrm{~min}$ to $75 \mathrm{~min}$ post stress. Those who reported negative social exchanges across relationships with roommates, family, and their closest friend showed greater IL- 6 responses to stress. Differences in mean IL- 6 were accounted for by either depressed mood or hostility, whereas differences in the cytokine stress responses remained significant after controlling for those factors. Overall, this research provides preliminary evidence to suggest that having multiple negative relationships may exacerbate acute inflammatory responses to a laboratory stressor independent of hostility and depressed mood. 
Running head: cytokine stress responses and social relationships

The role of multiple negative social relationships in inflammatory cytokine responses to a laboratory stressor

Sunmi Song ${ }^{1}$, Jennifer E. Graham-Engeland ${ }^{2}$, Elizabeth J. Corwin ${ }^{3}$, Rachel M. Ceballos ${ }^{4}$, Shelley E. Taylor $^{5}$, Teresa Seeman ${ }^{6}$, and Laura Cousino Klein ${ }^{7}$

${ }^{1}$ Social Behavioral Research Branch, National Human Genome Research Institute, National Institutes of Health, USA

${ }^{2}$ Department of Biobehavioral Health, The Pennsylvania State University, USA

${ }^{3}$ Nell Hodgson Woodruff School of Nursing, Emory University, USA

${ }^{4}$ Cancer Prevention Program, Fred Hutchinson Cancer Research Center, USA

${ }^{5}$ Department of Psychology, University of California, Los Angeles, USA

${ }^{6}$ Division of Geriatrics, David Geffen School of Medicine, University of California, Los Angeles, USA

${ }^{7}$ Department of Biobehavioral Health and Penn State Institute of the Neuroscience, The Pennsylvania State University, USA

*Correspondence should be addressed to Laura Cousino Klein, 219 Biobehavioral Health Building, The Pennsylvania State University, University Park, PA 16802, USA; E-mail: 1cklein@psu.edu. 
Abstract

37 The present study examined the unique impact of perceived negativity in multiple social relationships

38 on endocrine and inflammatory responses to a laboratory stressor. Via hierarchical cluster analysis,

39 those who reported negative social exchanges across relationships with a romantic partner, family, and

40 their closest friend had higher mean IL-6 across time and a greater increase in TNF- $\alpha$ from $15 \mathrm{~min}$ to

$4175 \mathrm{~min}$ post stress. Those who reported negative social exchanges across relationships with roommates,

42 family, and their closest friend showed greater IL-6 responses to stress. Differences in mean IL-6 were

43 accounted for by either depressed mood or hostility, whereas differences in the cytokine stress

44 responses remained significant after controlling for those factors. Overall, this research provides

45 preliminary evidence to suggest that having multiple negative relationships may exacerbate acute

46 inflammatory responses to a laboratory stressor independent of hostility and depressed mood.

47

48 Keywords: multiple negative social relationships; stress; inflammatory cytokine response; hostility;

49 depressed mood 
51 The role of multiple negative social relationships in inflammatory cytokine responses to a laboratory stressor

Stress is a routine part of daily life and interpersonal stress is often the most common and

54 arguably the strongest type of stressor most people experience (Kiecolt-Glaser, Gouin, \& Hantsoo,

55 2010). Poorer overall health and dysregulated immune function are strongly linked with interpersonal

56 stress both from negative social exchanges (Chiang, Eisenberger, Seeman, \& Taylor, 2012; Edwards,

57 Hershberger, Russell, \& Markert, 2001; Kiecolt-Glaser \& Newton, 2001) and chronic social conflict

58 (Cohen et al., 1998; Davis et al., 2008; Friedman, 2010). Interpersonal stress appears to have a long

59 lasting impact on health in part by contributing to chronically elevated inflammation, which can confer

60 risk of diverse age-related diseases (Ershler \& Keller, 2000; Graham, Christian, \& Kiecolt-Glaser,

612007 ; Ridker, 2000). However, the majority of studies on immune responses to social conflict have

62 focused on a particular type of relationship (e.g., marital relationships), while the effect of having

63 conflict across multiple relationships is largely unknown. Further, the degree to which multiple social

64 conflicts affect inflammatory responses to stress and whether the association is independent of related

65 psychosocial characteristics are important issues that are not well understood. The present research is

66 expected to advance the literature by examining interpersonal relationships in multiple areas and how

67 negativity across multiple interpersonal relationships affects inflammatory responses to a laboratory

68 stressor.

Studies most relevant to the current research have examined the effects of acute social conflict on health related outcomes. For example, the frequency of negative social exchanges with close others

71 has been negatively associated with physical and mental health among college students (Edwards et al.,

72 2001) and is predictive of depressed mood in a sample of married adults (Joiner \& Timmons, 2009).

73 Complementing such research, experimental studies have demonstrated that acute social conflict can

74 influence immune responses in a laboratory setting (Chiang et al., 2012; Kiecolt-Glaser et al., 2005). 
75 One mechanism that may explain the negative effects of acute social conflict on health is repeated

76 physiological activation of inflammatory stress responses and delayed recovery to stress (Seeman \&

77 McEwen, 1996). Under social conflict, inflammatory responses to stress may be also maintained by

78 actions of the sympathetic-adrenal-medullary (SAM) system and the hypothalamic-pituitary-adrenal

79 (HPA) axis (e.g., via cortisol) (Lovallo, 2005; Miller, Chen, \& Zhou, 2007). Recent studies show that

80 gene expression of inflammatory pathways are upregulated in leukocytes among socially stressed

81 individuals compared to matched control with good social support (Cole, Hawkley, Arevalo, \&

82 Cacioppo, 2011; Slavich \& Cole, 2013).

In addition to a relatively direct effect of social conflict via stress activation, it is important to

84

85

86

87

consider individual characteristics that tend to go along with negative social relationships. In particular, trait hostility and depressive symptoms appear to aggravate the effects of psychosocial stressors on cardiovascular and inflammatory response (Brondolo et al., 2003; Brummett et al., 2010). However, despite the possibility for hostility and depressed mood to be confounded with relationship stress and health, few relevant studies have controlled for hostility or depressed mood.

The present research aims to examine effects of negative social exchanges in multiple relationships - with a romantic partner, a close friend, close family members, and roommates - on responses to an experimental stressor, with an emphasis on inflammatory responses. We classified individuals into groups by their patterns of negative social exchanges across those four relationship areas. Because those who experience social conflict in many close relationships are a minority (Fingerman, Hay, \& Birditt, 2004), we expected to identify only a small number of those who reported negative social exchanges in multiple areas of measured relationships. We hypothesized that individuals with high levels of negative social exchanges in more relationships than others would have exaggerated or prolonged inflammatory response to stress (i.e., poorer recovery) in terms of two cytokines: IL-6 and TNF- $\alpha$. TNF- $\alpha$ is a classic proinflammatory cytokine and IL-6, although it has 
99 anti-inflammatory actions in certain contexts (for reviews see Hawkley, Bosch, Engeland, Marucha, \&

100 Cacioppo, 2007; Woods, Vieira, \& Keylock, 2009), is widely considered proinflammatory in the

101 context of psychological stress. We examined whether the effects of negative relationships on

102 inflammatory responses to stress are independent of depression or hostility. On a more exploratory

103 basis, we also expected that individuals with more negative relationships would show greater increases

104 in cortisol responses to the stressor.

105 Method

106 Participants

107 Fifty-six healthy participants ( 36 women, 20 men), aged $18-30$ years $($ mean $=21.05 \pm 0.37)$

108 were recruited to participate in a larger study examining influences of sex hormones to physiological

109 responses to an experimental stressor. Participants were recruited via advertisements in the local

110 newspaper and flyers posted in the local community and on the campus of a state university in the

111 Northeastern U.S. An initial telephone interview was conducted by a trained research assistant to

112 determine the eligibility of participants. Exclusion criteria included tobacco use, BMI $\geq 30$, psychiatric

113 hospitalization within the past year, the use of psychotropic medication, anti-inflammatory medications,

114 hormonal contraceptives, medications for controlling blood pressure, and inhaled beta agonists. People

115 who scored higher than the clinical cut off score of 16 on the Center for Epidemiologic Studies

116 Depression Scale (Radloff, 1977) or with history of depression were not eligible for the larger study. In

117 addition, we screened out people with a history of heart disease, diabetes, and neurological disorders.

118 Women who reported any possibility for pregnancy and menstrual cycle dysregulation also were

119 excluded. Women came to the laboratory during either the late luteal $(n=19)$ or follicular $(n=17)$

120 phase of their menstrual cycle for the purpose of the larger study.

\section{Measures}

122 Negative Social Relationships 
A 25-item measure was used to assess negative social relationships, which was based on an

124 existing questionnaire that included five items about negative social interactions with a spouse or

125 significant other (Schuster, Kessler, \& Aseltine Jr, 1990). The items ask about the frequency of

126 negative social exchanges involving disagreements, criticism, and tension, with responses ranging from

1270 (never) to 5 (very often). The present study used those same five items to ask participants about

128 negative social interactions among a) roommates, b) a romantic partner, c) close family members, d)

129 their closest friend, and e) their children. The alpha reliability of the original scale was 0.76 (Schuster

130 et al., 1990), and the present sample showed Chronbach- $\alpha$ of $0.84,0.89,0.84,0.81$ for the romantic

131 partner, family, roommate, and the closest friend subscales, respectively. As no participants reported

132 having children, that subscale was not used.

133 Negative Mood

134 A six item negative mood scale was administered four times (baseline, immediately after,

$13515 \mathrm{~min}$, and $75 \mathrm{~min}$ after the stressor) to check the effect of the experimental stressor on mood. The

136 scale consisted of words describing negative and positive mood (e.g., nervous, happy, irritated) with a

137 7-point Likert scale ranging from 1 (Not at all) to 7 (Very much). The scale showed a good internal

138 consistency across measurements (Chronbach- $\alpha=0.74,0.88,0.84$, and 0.86, respectively).

139 Depressed mood

140 The Center for Epidemiological Studies Depression Scale (CES-D; Radloff, 1977) was used to

141 measure depressed mood, which effectively identifies depressed mood among healthy individuals

142 (Radloff, 1977). Item responses are from 0 to 3, with 3 representing the greatest frequency of depressed

143 symptoms over the past week. The CES-D showed a chronbach- $\alpha$ of .90 for this sample.

\section{Hostility}

145 The well-validated Cook-Medley hostility questionnaire (CMHQ; Cook \& Medley, 1954) was

146 used to assess the tendency to react and think in a hostile manner. The scale has 50 true-false items, 
147 which are aggregated into a total score ranging from 0 to 50 . The chronbach- $\alpha$ was .83 for this sample.

148 Procedures

149 Laboratory Protocol and Stressor

150 Eligible participants arrived at a General Clinical Research Center (GCRC) at $1300 \mathrm{hrs}$ and

151 were met by a trained research assistant who first obtained a written informed consent. Next,

152 participants were interviewed by a certified nurse practitioner to confirm health status and study

153 eligibility. Participants then were asked to complete questionnaires, after which a trained nurse inserted

154 an indwelling catheter in the non-dominant arm. After a 10min acclimation period, participants were

155 asked to sit quietly for $15 \mathrm{~min}$. A baseline blood sample (20cc) was then drawn.

Next, a modified Trier Social Stress Task was administered. Participants were given 10min to

prepare a $3 \frac{1}{2}$-minute speech about a personal failure that had a negative consequence on their life.

They delivered the speech in front of a video camera and were told that a recording would be later

observed by a panel of psychologists (no recording was actually made). Participants were prompted by

the experimenter to continue talking if they finished their speech in less than the allotted time.

161 Immediately after the speech, participants were asked to complete a serial subtraction task as fast and

162 as accurately as possible (4min), followed by several math word questions that increased in difficulty

163 (3.5 $\mathrm{min})$, and then another serial subtraction task (4min). The experimenter delivered timed prompts to

164 urge participants to work more quickly and to tell them to start over if they delivered the wrong

165 response. This stress protocol took $30 \mathrm{~min}$ total.

Baseline blood samples and blood samples at 15 and $75 \mathrm{~min}$ after the stress period were used to

167 determine cortisol, IL-6, and TNF- $\alpha$. Participants completed several post-stress measures of mood at

168 the end of the study, after which the catheter was removed. The study procedure was approved by the

169 institutional review board at the Pennsylvania State University (IRB \# 00M0314-B9).

170 Blood Handling 
For preparation of serum, blood was drawn into separate collection tubes that contained no

172 additive. Serum tubes were allowed to sit at room temperature for $15 \mathrm{~min}$ before centrifugation $(1500 \times$

$173 \mathrm{~g}$ at $4{ }^{\circ} \mathrm{C}$ for 15 minutes). Following centrifugation, serum was aliquoted into separate $100 \mu \mathrm{L}$

174 microtubes and frozen at $-80^{\circ} \mathrm{C}$ for later assay.

175 Serum Cortisol, IL-6, and TNF- $\alpha$

Assays were performed at the Pennsylvania State University GCRC Core Laboratory. Serum

177 cortisol levels were determined using commercially available enzyme immunoassay kits (EIA;

178 Diagnostic Systems Laboratories, Inc., Webster, TX). The inter-assay and intra-assay coefficients of

179 variation were $3.16 \%$ and $4.8 \%$, respectively for cortisol. Serum IL-6 and TNF- $\alpha$ levels were

180 determined by enzyme-linked immunosorbant assays constructed with antibodies purchased from R\&D

181 systems (Minneapolis, MN) using previously described procedure (Corwin, Bozoky, Pugh, \& Johnston,

182 2003). The level of detection was $1.0-3.0 \mathrm{pg} / \mathrm{mL}$, and inter-assay and intra-assay coefficients of

183 variation were $7.1 \%$ and $5.3 \%$ respectively for these cytokine assays. All samples were tested in

184 duplicate in a single assay batch; values that varied by more than $5 \%$ were subject to repeat testing.

185 The average of duplicate tests is reported for each biomarker assay.

\section{Data Analysis}

SPSS 20.0 was used for all analyses. Study variables were screened for outliers and nonnormality, and cortisol, IL-6, and, TNF- $\alpha$ were natural log transformed to correct for skewness. Next, a hierarchical cluster analysis was applied to classify individuals with different patterns of perceived negativity across the relationships with roommates, a romantic partner, close family, and a closest

191 friend. Out of 56 participants, one woman did not provide sufficient data to compute the cluster by

192 negative relationship analyses and was therefore excluded from analyses. While 25 participants

193 reported having all of the four relationships, 13 participants did not have a roommate, and 13 others did 194 not have a romantic partner. Thus, two separate hierarchical cluster analyses were run, the first cluster 
195 analysis including those who reported having a romantic partner, a closest friend, and family $(n=38)$

196 and the second including individuals who reported having roommates, a closest friend, and family $(n=$

197 38). Four participants who did not report a relationship with either a romantic partner or roommates

198 were excluded from these cluster analyses; there was no significant difference between those four

199 participants and the rest of the sample in any psychological characteristics or outcome variable we

200 examined.

201 In each of two cluster analyses conducted, the same three steps were used. First, the variables

202 for the perceived negativity in the three relationship areas were entered via hierarchical cluster analysis.

203 Ward's method with the similarity measure of squared Euclidean distance was then used to decide the

204 number of groups in the cluster model. Discriminant function analysis was used to verify how much of

205 the clustering within groups could be replicated (Klecka, 1980). In the third step, we further examined

206 the characteristics of the groups in the cluster model via $F$ tests by examining whether the groups

207 differed by age, gender, and the four negative relationship variables.

208 The general linear model (GLM) with within-subject design was then used to examine the

209 effect of the different negative social relationship clusters on cortisol and cytokine responses to stress.

210 Greenhouse-Geisser correction was used for sphericity. For the significant results, the partial eta-

211 squared $\left(\eta p^{2}\right)$ post hoc tests with Bonferroni correction were reported. As post hoc tests for the time

212 effects, difference scores were calculated for the stress response measures between each pair of the

213 three time points (e.g., from baseline to $15 \mathrm{~min}$ after stress) in order to examine change during each

214 time interval. Due to their known impact on inflammation, age, BMI, gender, and menstrual cycle of

215 women were controlled in all of these analyses. Three dummy coded variables representing a) men, b)

216 women in the luteal period, and c) women in the follicular period were generated and entered in

217 analyses to control for both gender and women's menstrual cycle status. Finally, depressed mood and

218 hostility were additionally entered to the GLM to examine whether the effects were independent of 
219 those characteristics.

220 Results

\section{Preliminary analyses}

The means and standard deviations (SDs) of demographics and study variables are presented in

223 Table 1. The sample was predominantly Caucasian (71\%) and comprised of young adults with a mean 224 age of $21.05(\mathrm{SD}=2.74$, range $18-30)$.

\section{Cluster analysis for negative relationship profiles}

We classified individuals as having different degrees of negative social exchanges across

227 multiple interpersonal relationship areas. The first cluster analysis was run on the 38 participants who 228 had a romantic partner, family, and a close friend. It yielded two groups (Table 2, a): "a low conflict

229 group" $(n=29)$ characterized by consistently low levels of negative social exchanges across all

230 relationship areas (romantic partner, family, and their closest friend), and "a multiple conflict group" (n

$231=9$ ) that had high levels of negative social exchanges across all relationship areas. $F$ tests confirmed

232 that the two groups in this cluster model were different in levels of negative social exchanges in these

233 relationships $(p s<.05)$. A discriminant function analysis verified the cluster structure $\left(\chi^{2}(3, n=38)=\right.$

$23453.56, p<.001$ ), and 97.4\% of the original grouped cases (37 cases out of 38) were replicated. The

235 distribution of gender and age was not significantly different across the two groups.

236 Another cluster analysis was run on the 38 participants who reported relationships with

237 roommates, family, and the closest friend. This analysis identified three clusters (Table 2, b): “a low

238 conflict group" $(n=30)$ characterized by low levels of negative social exchanges across all the

239 relationship areas, "a multiple conflict group" $(n=3)$ characterized by high levels of negative social

240 exchanges across all the relationship areas, and "a family conflict group" $(n=5)$ characterized

241 primarily by a high level of negative social exchanges in family but low levels of negative social

242 exchanges among roommates and the closest friend. The $F$ tests confirmed that the three groups in this 
243 cluster analysis (hereafter referred to as the "roommate model") were different in levels of negative

244 social exchanges across the three relationship areas $(p s<.001)$. A discriminant function analysis

245 verified the three cluster structure $\left(\chi^{2}(6, n=38)=70.69, p<.001\right)$ and that $94.7 \%$ (36 cases out of 38$)$

246 of the original grouped cases were replicated. The gender distribution was significantly different across

247 the 3 groups $(p=.05)$, which was largely driven by the multiple conflict group having only three men

248 and no women. Age was not different across the 3 groups.

249 Manipulation checks for the stress protocols

250 Participants' negative mood increased in response to the stressor $(F(3,153)=34.71, p<.001$,

$\left.251 \eta p^{2}=.28\right)$. The levels of serum cortisol did not significantly increase in response to the experimental

252 stressor but showed a significant decrease over time $\left(F(2,106)=9.88, p<.001, \eta p^{2}=.16\right)$, likely

253 driven primarily by the diurnal rhythm of cortisol. There was no significant time effect on IL-6 and

254 TNF- $\alpha$ levels.

255 Baseline differences in biomarkers by negative social relationships

The Table 1 presents the baseline levels of biomarkers by cluster groups in both the romantic

257 partner model and the roommate model. There was a significant baseline difference in IL-6 levels

258 between the low and multiple conflict groups in romantic partner model $\left(F(1,36)=4.18, p=.05, \eta p^{2}=\right.$

259 .10). The baseline difference became not significant after controlling for depression or hostility along

260 with age, gender, and menstrual cycle status. There were no other baseline differences in any of the

261 groups in either the romantic partner or roommate model.

262 Stress responses by negative social relationships

$263 \quad I L-6$

There was a significant time by negative social relationship interaction using the roommates

265 model on IL-6 $\left(F(4,58)=8.53, p<.01, \eta p^{2}=.37\right)$. Post hoc tests confirmed that only individuals in

266 the multiple conflict group of this cluster model showed significantly greater increases in IL-6 from 
267 baseline to $15 \mathrm{~min}$ after stress $(p s<.01)$ and from baseline to $75 \mathrm{~min}$ after stress $(p s<.01)$ compared to

268 those in the family conflict or low conflict groups (Figure 1). Results remained significant after

269 controlling for depressed mood or hostility $(p s<.01)$.

$270 \quad T N F-\alpha$

There was a marginally significant time by negative social relationship interaction among the

romantic partner model on TNF- $\alpha$ responses to the stressor $\left(F(2,56)=2.80, p=.07, \eta p^{2}=.09\right)$. Upon

273

examination, individuals in the multiple conflict group (who reported negativity in their relationships

with their romantic partner as well as their closest friend, and family) showed significantly greater increases in TNF- $\alpha$ from $15 \mathrm{~min}$ to $75 \mathrm{~min}$ post stress after controlling for baseline TNF- $\alpha$ and other

covariates $\left(F(1,27)=6.81, p<.05 \eta p^{2}=.20\right)$, as illustrated in Figure 2. This remained significant after controlling for depressed mood or hostility $(p s<.05)$ and also after removing the one overlapping individual in the multiple conflict group who was also included in the multiple conflict group in the roommate model (where greater IL-6 responses to stress were observed). There was no main effect in the roommate model groups on TNF- $\alpha$ stress response.

\section{Cortisol}

Neither of the negative relationship cluster models significantly predicted cortisol responses to 283 the stressor.

\section{Discussion}

Although social conflict has been associated consistently with poorer health and various stressrelated biomarkers (Graham et al., 2007; Kiecolt-Glaser \& Newton, 2001), the majority of past studies showing connections between relationship stress and biomarkers have focused on a particular type of relationship or broad characterizations of relationship quality or network size. The present research is the first to examine the effect of negative social exchanges across multiple interpersonal relationships 290 and whether the effects of negative social exchanges in multiple relationship areas are independent of 
291 depressed mood and trait hostility. Another novel aspect of the present research is that we focused on

292 the effect of relationships on inflammatory responses to stress as opposed to basal levels of biomarkers.

293 As expected, the present results suggest that there are differences in acute inflammatory cytokine

294 responses to stress depending on the pattern of multiple negative social relationships individuals

295 reported within the four relationship areas examined (romantic partner, family, the closest friend, and

296 roommates). Those who reported negative social exchanges in their relationships with roommates,

297 family, and their closest friend showed increases in IL-6 after being exposed to a laboratory stressor.

298 Similarly, people who reported negative social exchanges with a romantic partner, family, and their

299 closest friend showed increases in TNF- $\alpha$ from $15 \mathrm{~min}$ to $75 \mathrm{~min}$ post stress after controlling for

300 baseline TNF- $\alpha$. Both the IL- 6 and the TNF- $\alpha$ results remained significant after controlling for

301 depressed mood, hostility, age, BMI, gender, and menstrual cycle status.

302 Importantly, the two multiple conflict groups examined in this research (among those who had

303 roommates, vs. those who had a romantic partner) were largely distinct from each other; there was only

304 one individual who was included in both of these analyses. Thus, we showed an effect of negativity

305 across multiple relationship areas on increases in either TNF- $\alpha$ or IL-6 via different subsets of

306 individuals, suggesting that the effects of having social conflict across relationship areas are robust and

307 pro-inflammatory in nature. Further, results were not driven by baseline effects in inflammation or

308 outliers. We did not find any outliers in either of the groups that showed significant stress responses

309 and in all participants. Further, as compared to the other groups, individuals in the multiple conflict

310 group of the roommate model did not evidence significantly different baseline levels of IL-6 and those

311 in the multiple conflict group of the romantic partner model did not evidence significantly different

312 baseline levels of TNF- $\alpha$. Thus, participants in those two multiple conflict groups came to the lab

313 without elevated IL- 6 or TNF- $\alpha$ compared to others, but were the ones who showed increases in IL-6

314 or TNF- $\alpha$ after being exposed to the laboratory stressor. 
The findings of the present research complement the results of a recent study showing that daily

316 levels of negative social interactions were associated with greater inflammatory responses to an

317 experimental stressor as measured by IL-6 and soluble TNF- $\alpha$ receptor II (Chiang et al., 2012). In

318 terms of direction, the inflammatory stress reactivity of individuals with multiple negative relationships

319 is also consistent with previous studies of the association between a laboratory induced social conflict

320 and inflammatory responses (Graham et al., 2007; Kiecolt-Glaser et al., 2005). Psychological stress

321 effects on excessive inflammatory cytokines responses are likely explained by multiple aspects of

322 complex, interrelated physiological systems. For example, chronic interpersonal stress is likely be

323 related to dysregulation of the inflammatory stress response due to decreased glucocorticoid receptor

324 sensitivity (Corwin et al., 2013; Miller et al., 2007; Pace, 2012) or perhaps to down-regulation of

325 cholinergic anti-inflammatory pathways in neural circuitry (Tracey, 2002).

Interestingly, there was one baseline difference in IL-6 observed: Participants who reported multiple conflict across relationships and who had a romantic partner had higher IL-6 at baseline, an

328 effect that was reduced to non-significance when controlling for either depressed mood or hostility and

329 which was not observed among those who reported multiple relationship conflict and who had

330 roommates. It may be that for this relatively young sample, which was largely comprised of college

331 students $(78 \%)$, that those who were in conflictive romantic relationships were more depressed or

332 hostile and that this (rather than the conflict itself) explained their higher baseline IL-6. In contrast,

333 those in the present study who had conflicts with roommates may have had less control over their

334 exposure to those individuals and may have developed greater inflammatory stress reactivity due to

335 frequent exposure to stress. The difference observed between those with roommates and romantic

336 partners was not expected, and may be limited to the particular sample in the present research.

337 However, the finding that depressed mood and hostility can explain baseline levels of inflammation is

338 consistent with previous research showing that depressed mood and hostility are associated with 
339 increased circulating markers of inflammation among adults (Graham, Christian, \& Kiecolt-Glaser,

340 2006; Suarez, Lewis, \& Kuhn, 2002; Zorrilla et al., 2001). Importantly, no such baseline effects

341 explained the effects of relationship negativity on the inflammatory cytokine responses to acute stress

342 observed in the present research.

343 We did not find a significant effect of negative relationship endorsement on cortisol responses.

344 This null effect might be related to the experimental stress paradigm used in which the public speech

345 part of the stress task was conducted in front of a video camera instead of a panel of judges, a protocol

346 which can reduce the intensity of stress response from the Trier (Dickerson \& Kemeny, 2004;

347 Kirschbaum, Pirke, \& Hellhammer, 1993). Also, the timing of the catheter insertion and blood draws

348 might also explain the null finding.

$349 \quad$ Limitations

350 The clinical implications of the study are limited in several ways. The present research was

351 conducted with a small sample of healthy, relatively young adults, which limits generalizability and

352 warrants cautions in interpretation. However, a relatively small proportion of a sample can be expected

353 to have multiple relationship conflicts and the issues with there being a small number of those with

354 multiple conflicts were minimized in the present research by our application of conservative statistical

355 adjustments when using cluster analysis and general linear model and comparisons of analyses between

356 the two multiple conflict clusters (Clatworthy, 2005; Hair, Tatham, Anderson, \& Black, 2006; Huynh,

357 1978). It will be important to replicate the present results using larger and more diverse samples,

358 particularly a greater number of individuals reporting negative relationships across multiple social

359 relationship areas than was available in the present study. It was also a limitation that we only had IL-6

360 and TNF- $\alpha$ available as inflammatory biomarkers: Future research on related topics would benefit from

361 utilizing multiplex technology to examine a greater diversity of analytes, including anti-inflammatory

362 cytokines (e.g., IL-10) to enable examination of the ratio between pro and anti-inflammatory cytokines 
363 in stress responses. It would also have been ideal if we had been able to include blood draws later than

$36475 \mathrm{~min}$ post-stress to capture the full peak as well as return to baseline of the cytokine responses.

365 Finally, it would be of value for future research to utilize clinically diverse samples, such as those with

366 clinical depression or existing inflammatory conditions, and to include assessments of clinical health

367 outcomes.

368 Conclusion

369 A better appreciation for how social conflict may alter stress responsiveness of the body is

370 critical to understanding how and why it is associated with poorer physical health (Seeman \& McEwen,

371 1996). Although having some degree of social conflict is an unavoidable part of everyday life, the

372 present study provides preliminary evidence that having negative social interactions across multiple

373 social relationships might be harmful, as it is associated with greater inflammatory reactivity to a

374 psychosocial stressor. Significant increases in both IL-6 and TNF- $\alpha$ in response to stress were observed

375 among those with relationship conflict in at least three areas, compared to those with relationship

376 conflict in fewer relationships. The effect of having multiple social relationships on inflammatory

377 responses to stress appears to be independent of any effect of hostility or depressed mood. Taken as a

378 whole, the present research emphasizes the importance of examining the role of negative close

379 relationships in inflammatory stress response in a detailed fashion. Having multiple negative

380 relationships may put individuals at particular risk of developing exacerbated acute inflammatory

381 reactivity to psychosocial stress.

384 Acknowledgements

385 We appreciate the dedicated assistance of undergraduate students in the Biobehavioral Health Studies

386 Lab, the GCRC nursing staff, M.M. Stine and C.A. Whetzel. 
388 Funding Statements

389 This research was conducted at The Pennsylvania State University. The services provided by the 390 General Clinical Research Center of The Pennsylvania State University are appreciated (NIH grant 391 MO1-RR-10732). Funding was provided by the NSF (SBR9905157; SET and LCK) and a grant from

392 the College of Health and Human Development of The Pennsylvania State University (223-15-3605;

393 LCK and EJC).

394 
396 Brondolo, E., Rieppi, R., Erickson, S. A., Bagiella, E., Shapiro, P. A., McKinley, P., \& Sloan, R. P. (2003). Hostility, interpersonal interactions, and ambulatory blood pressure. Psychosomatic Medicine, 65(6), 1003-1011.

Brummett, B. H., Boyle, S. H., Ortel, T. L., Becker, R. C., Siegler, I. C., \& Williams, R. B. (2010). Associations of depressive symptoms, trait hostility, and gender With C-reactive protein and interleukin-6 response after emotion recall. Psychosomatic Medicine, 72, 333-339.

Chiang, J. J., Eisenberger, N. I., Seeman, T. E., \& Taylor, S. E. (2012). Negative and competitive social interactions are related to heightened proinflammatory cytokine activity. Proceedings of the National Academy of Sciences of the United States of America, 109, 1878-1882.

Clatworthy, J., Buick, D., Hankins, M., Weinman, J., \& Horne, R. (2005). The use and reporting of cluster analysis in health psychology: A review. British Journal of Health Psychology, 10, 329358.

Cohen, S., Frank, E., Doyle, W. J., Skoner, D. P., Rabin, B. S., \& Gwaltney, J. M. (1998). Types of stressors that increase susceptibility to the common cold in healthy adults. Health Psychology, $17,214-223$.

Cole, S. W., Hawkley, L. C., Arevalo, J. M. G., \& Cacioppo, J. T. (2011). Transcript origin analysis identifies antigen-presenting cells as primary targets of socially regulated gene expression in leukocytes. Proceedings of the National Academy of Sciences, 108(7), 3080-3085. doi: 10.1073/pnas. 1014218108

Cook, W. W., \& Medley, D. M. (1954). Proposed hostility and pharisaic virtue scales for the MMPI. Journal of Applied Psychology, 38, 414-418.

Corwin, E. J., Bozoky, I., Pugh, L. C., \& Johnston, N. (2003). Interleukin-1beta elevation during the postpartum period. Annals of Behavioral Medicine, 25(1), 41-47. 
419 Corwin, E. J., Guo, Y., Pajer, K., Lowe, N., McCarthy, D., Schmiege, S., . . Stafford, B. (2013). Immune dysregulation and glucocorticoid resistance in minority and low income pregnant women. Psychoneuroendocrinology, 38(9), 1786-1796.

Davis, M. C., Zautra, A. J., Younger, J., Motivala, S. J., Attrep, J., \& Irwin, M. R. (2008). Chronic stress and regulation of cellular markers of inflammation in rheumatoid arthritis: Implications for fatigue. Brain, Behavior, and Immunity, 22, 24-32.

425 integration and synthesis of laboratory research. Psychological Bulletin, 130, 355-391.

Edwards, K. J., Hershberger, P. J., Russell, R. K., \& Markert, R. J. (2001). Stress, negative social exchange, and health symptoms in university students. Journal of American College Health, 50, 75-79.

Ershler, W. B., \& Keller, E. T. (2000). Age-associated increased interleukin-6 gene expression, latelife diseases, and frailty. Annual Review of Medicine, 51, 245-270.

Fingerman, K. L., Hay, E. L., \& Birditt, K. S. (2004). The Best of Ties, the Worst of Ties: Close, Problematic, and Ambivalent Social Relationships. Journal of Marriage and Family, 66, 792808.

Friedman, E. M., Karlamangla, A., Almeida, D. M., \& Seeman, T. E. (2010). Social conflict and cortisol regulation. California Center for Population Research: On-Line Working Paper Series, 1-38. http://papers.ccpr.ucla.edu/

Graham, J. E., Christian, L. M., \& Kiecolt-Glaser, J. (2006). Stress, Age, and Immune Function: Toward a Lifespan Approach. Journal of Behavioral Medicine, 29, 389-400.

Graham, J. E., Christian, L. M., \& Kiecolt-Glaser, J. K. (2007). Close relationships and immunity. In R. Ader (Ed.), Psychoneuroimmunology (pp. 781-798). Burlington, MA: Elsevier Academic Press. 
442 Hair, J. F., Tatham, R. L., Anderson, R. E., \& Black, W. (2006). Multivariate data analysis (Vol. 6): Pearson Prentice Hall Upper Saddle River, NJ.

444 Hawkley, L. C., Bosch, J. A., Engeland, C. G., Marucha, P. T., \& Cacioppo, J. T. (2007). Loneliness, 445 dysphoria, stress and immunity: A role of cytokine. In N. P. Plotnikoff, R. E. Faith, A. J. Murgo \& R. A. Good (Eds.), Cytokine: Stress and immunity (pp. 67-86). Boca Raton, FL: CRC press.

$$
\text { 161-175. }
$$

Joiner, T. E., \& Timmons, K. A. (2009). Depression in its interpersonal context. In I. H. Gotlib \& C. L. Hammen (Eds.), Handbook of Depression (2nd ed.). New York, NY: The Guildford Press.

Kiecolt-Glaser, J. K., Gouin, J.-P., \& Hantsoo, L. (2010). Close relationships, inflammation, and health. Neuroscience and Biobehavioral Reviews, 35, 33-38.

Kiecolt-Glaser, J. K., Loving, T. J., Stowell, J. R., Malarkey, W. B., Lemeshow, S., Dickinson, S. L., \& Glaser, R. (2005). Hostile marital interactions, proinflammatory cytokine production, and wound healing. Archives of General Psychiatry, 62, 1377-1384.

Kiecolt-Glaser, J. K., \& Newton, T. L. (2001). Marriage and health: His and hers. Psychological Bulletin, 127, 472-503.

Kirschbaum, C., Pirke, K. M., \& Hellhammer, D. H. (1993). The “Trier Social Stress Test”-A tool for investigating psychobiological stress responses in a laboratory setting. Neuropsychobiology, 28, $76-81$.

Klecka, W. R. (1980). Discriminant analysis. Newbury Park, CA: Sage Publications.

Lovallo, W. (2005) Stress and Health: Biological and Psychological Interactions (2nd ed., pp. 197205). Thousand Oaks, CA: Sage Publications.

Miller, G. E., Chen, E., \& Zhou, E. S. (2007). If it goes up, must it come down? Chronic stress and the hypothalamic-pituitary-adrenocortical axis in humans. Psychological Bulletin, 133, 25-45. 
466 Pace, T. W., Wingenfeld, K., Schmidt, I., Meinlschmidt, G., Hellhammer, D.H., \& Heim, C.M. (2012). Increased peripheral NF-kappa B pathway activity in women with childhood abuse-related posttraumatic stress disorder. Brain Behavior and Immunity, 26, 13-17.

469

470

471

472

473

474

475

476

477

478

479

480

481

482

483

484

485

486

Radloff, L. S. (1977). The CES-D Scale: A self-report depression scale for research in the general population. Applied Psychological Measurement, 1, 385-401.

Ridker, P., Rifai, N., Stampfer, M., \& Hennekens, C. (2000). Plasma concentration of interleukin-6 and the risk of future myocardial infarction among apparently healthy men. Circulation, 101, 17671772.

Schuster, T. L., Kessler, R. C., \& Aseltine Jr, R. H. (1990). Supportive interactions, negative interactions, and depressed mood. American journal of community psychology, 18, 423-438.

Seeman, T. E., \& McEwen, B. S. (1996). Impact of social environment characteristics on neuroendocrine regulation. Psychosomatic Medicine, 58, 459-471.

Slavich, G. M., \& Cole, S. W. (2013). The Emerging Field of Human Social Genomics. Clin Psychol Sci, 1(3), 331-348.

Suarez, E. C., Lewis, J. G., \& Kuhn, C. (2002). The relation of aggression, hostility, and anger to lipopolysaccharide-stimulated tumor necrosis factor (TNF)-alpha by blood monocytes from normal men. Brain, Behavior, and Immunity, 16, 675-684.

Tracey, K. J. (2002). The inflammatory reflex. Nature, 420, 853-859.

Woods, J. A., Vieira, V. J., \& Keylock, K. T. (2009). Exercise, inflammation, and innate immunity. Immunology and Allergy Clinics of North America, 29(2), 381-393.

Zorrilla, E. P., Luborsky, L., McKay, J. R., Rosenthal, R., Houldin, A., Tax, A., . . Schmidt, K. (2001). The Relationship of Depression and Stressors to Immunological Assays: A Meta-Analytic Review. Brain, Behavior, and Immunity, 15(3), 199-226. 


\section{Table $\mathbf{1}$ (on next page)}

Sample characteristics and the baseline measure of biomarkers by cluster groups

Note. Biomarker levels were log transformed; $\mu \mathrm{g} / \mathrm{dL}=$ micrograms per deciliter; $\mathrm{pg} / \mathrm{mL}=$ picograms per milliliter. 
2 Table 1. Sample characteristics and the baseline measure of biomarkers by cluster groups

\begin{tabular}{|c|c|c|c|c|}
\hline & \multicolumn{2}{|c|}{ Total $(\mathrm{N}=56)$} \\
\hline & & & $M$ & $S D$ \\
\hline \multicolumn{5}{|l|}{ Characteristics } \\
\hline \multicolumn{2}{|l|}{ Age (yrs) } & & 21.05 & 2.74 \\
\hline \multicolumn{2}{|l|}{ Women $(\%)$} & & 64.3 & \\
\hline \multicolumn{5}{|c|}{ Cycling status among women $(n=36)$} \\
\hline \multicolumn{2}{|l|}{ Luteal $(\%)$} & & 52.78 & \\
\hline \multicolumn{2}{|l|}{ Follicular (\%) } & & 47.22 & \\
\hline \multicolumn{2}{|c|}{ Body Mass Index $\left(\mathrm{kg} / \mathrm{m}^{2}\right)$} & & 23.42 & 3.10 \\
\hline \multicolumn{2}{|l|}{ Hostility } & & 23.32 & 6.85 \\
\hline \multicolumn{2}{|l|}{ Depressed Mood } & & 9.29 & 8.30 \\
\hline \multicolumn{5}{|c|}{ Baseline measure of biomarkers by cluster groups } \\
\hline Biomarker & Model & Groups & $M$ & $S D$ \\
\hline \multirow[t]{6}{*}{ Cortisol ( $\mu \mathrm{g} / \mathrm{dL})$} & Romantic partner model & Low conflict & 2.38 & 0.45 \\
\hline & & Multiple conflict & 2.23 & 0.43 \\
\hline & Roommate model & Low conflict & 2.38 & 0.42 \\
\hline & & Only family conflict & 2.02 & 0.37 \\
\hline & & Multiple conflict & 2.47 & 0.20 \\
\hline & & Total & 2.37 & 0.42 \\
\hline \multirow[t]{3}{*}{ IL-6 (pg/mL) } & Romantic partner model & Low conflict & 2.96 & 0.97 \\
\hline & & Multiple conflict & 3.74 & 1.15 \\
\hline & Roommate model & Low conflict & 3.41 & 1.40 \\
\hline
\end{tabular}




\begin{tabular}{|c|c|c|c|c|}
\hline & & Only family conflict & 4.25 & 0.78 \\
\hline & & Multiple conflict & 3.95 & 1.10 \\
\hline & & Total & 3.46 & 1.23 \\
\hline \multirow[t]{6}{*}{$\mathrm{TNF}-\alpha(\mathrm{pg} / \mathrm{mL})$} & Romantic partner model & Low conflict & 3.07 & 0.93 \\
\hline & & Multiple conflict & 3.31 & 1.08 \\
\hline & Roommate model & Low conflict & 3.44 & 1.21 \\
\hline & & Only family conflict & 4.18 & 1.27 \\
\hline & & Multiple conflict & 3.79 & 0.78 \\
\hline & & Total & 3.42 & 1.11 \\
\hline
\end{tabular}

3 Note. Biomarker levels were log transformed; $\mu \mathrm{g} / \mathrm{dL}=$ micrograms per deciliter; $\mathrm{pg} / \mathrm{mL}=$ picograms 4 per milliliter. 
Table 2 (on next page)

The level of negative social exchanges in each relationship area for the cluster groups, generated (a) by relationships with a romantic partner, family, and the closest friend and (b) by relationships with roommates, family, and the closest friend 
2 Table 2. The level of negative social exchanges in each relationship area for the cluster groups,

3 generated (a) by relationships with a romantic partner, family, and the closest friend and (b) by

4 relationships with roommates, family, and the closest friend

$5 \quad$ (a)

\begin{tabular}{|c|c|c|c|c|}
\hline \multirow{3}{*}{$\begin{array}{l}\text { Negative social } \\
\text { exchanges in the } \\
\text { relationships with }\end{array}$} & \multicolumn{4}{|c|}{ The Romantic Partner Model } \\
\hline & \multicolumn{2}{|c|}{$\begin{array}{l}\text { Low conflict } \\
\qquad(n=29)\end{array}$} & \multicolumn{2}{|c|}{$\begin{array}{l}\text { Multiple conflict } \\
\qquad(n=9)\end{array}$} \\
\hline & $M$ & $S D$ & $M$ & $S D$ \\
\hline Romantic partner & 7.86 & 2.45 & 11.89 & 4.91 \\
\hline Family & 8.07 & 2.15 & 16.44 & 1.88 \\
\hline The closest friend & 6.72 & 2.10 & 8.89 & 3.92 \\
\hline
\end{tabular}

7 (b)

The Roommates Model

Negative social

exchanges in the

Low conflict $\quad$ Only family conflict $\quad$ Multiple conflict

relationships with

$$
(n=30) \quad(n=5) \quad(n=3)
$$

\begin{tabular}{llllll}
\hline$M$ & $S D$ & $M$ & $S D$ & $M$ & $S D$
\end{tabular}

\begin{tabular}{lllllll}
\hline Roommates & 8.60 & 3.40 & 6.60 & 2.30 & 17.00 & 3.46
\end{tabular}

\begin{tabular}{lcccccc}
\hline Family & 8.60 & 2.75 & 17.60 & 1.95 & 20.33 & 2.52 \\
\hline The closest friend & 6.53 & 1.89 & 7.80 & 3.03 & 16.00 & 1.73
\end{tabular}


Figure 1 (on next page)

Changes in serum IL- 6 levels by the negative relationship groups in the roommate model

* Note. LN = Natural Log transformation 


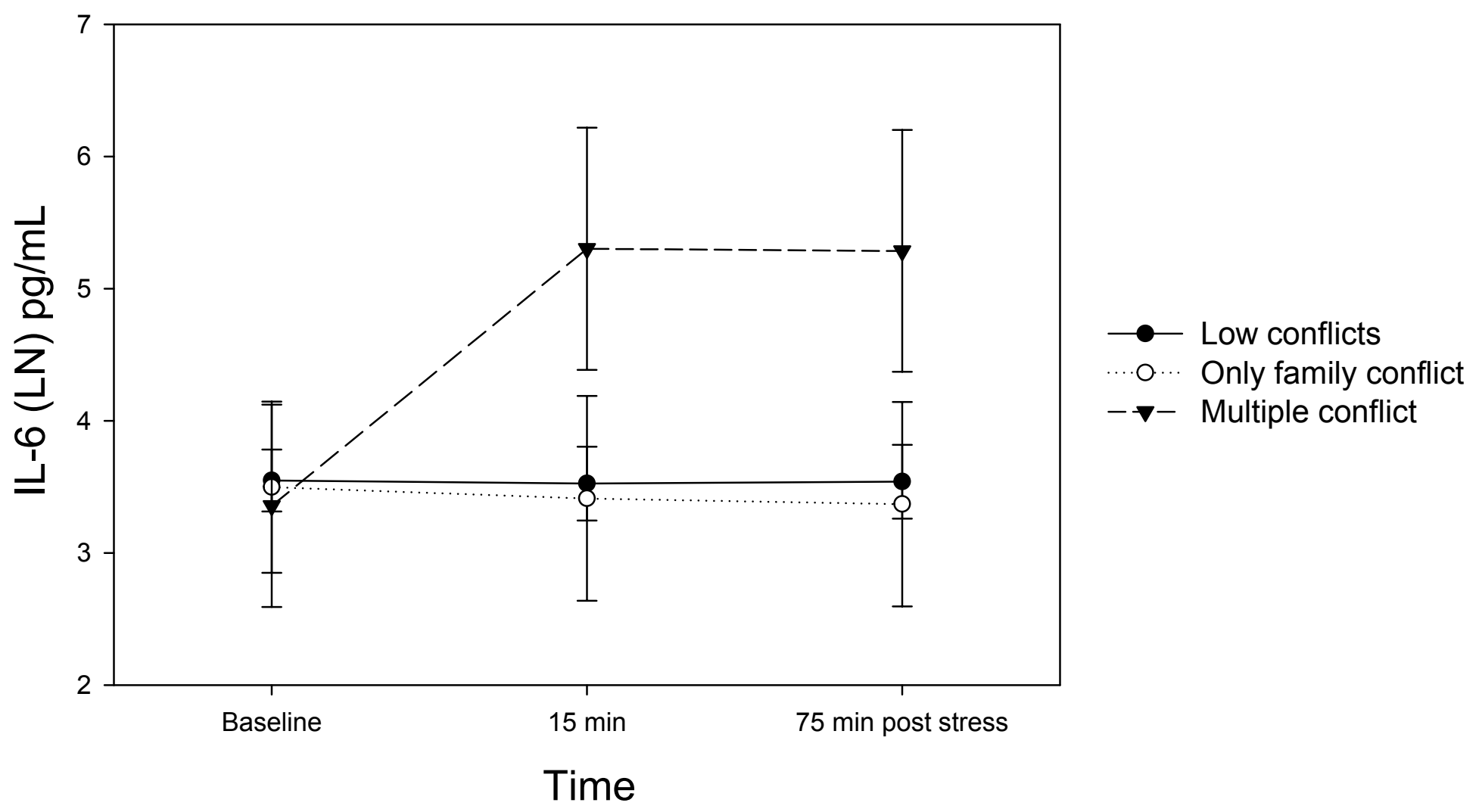


Figure 2 (on next page)

Changes in serum TNF- $\alpha$ levels by the negative relationship groups in the romantic partner model

* Note. LN = Natural Log transformation 


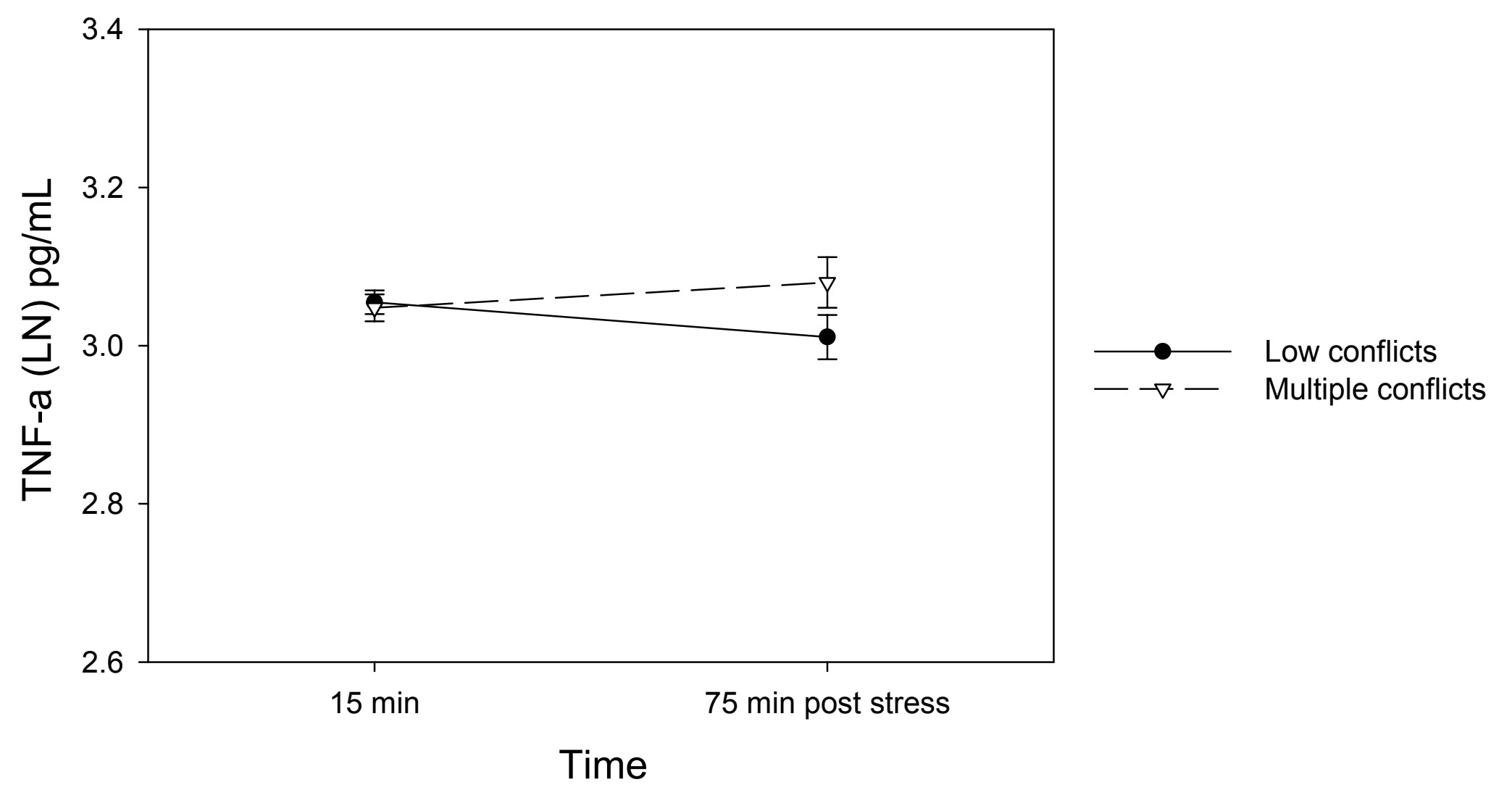

